\title{
Correlation of radio- and histomorphological pattern of pulmonary adenocarcinoma
}

\author{
Mathieu Lederlin*,\#,ף, Michael Puderbach\#, Thomas Muley ${ }^{+}$, Philipp A. Schnabel ${ }^{\S}$, \\ Albrecht Stenzinger ${ }^{\xi}$, Hans-Ulrich Kauczor*, Claus Peter Heussel ${ }^{\#}$, Felix J. F. Herth ${ }^{f}$, \\ Hans Hoffmann**, Hendrik Dienemann**, Wilko Weichert ${ }^{\S, \# \#}$ and Arne Warth ${ }^{\S, \# \#}$
}

ABSTRACT: Recently, a novel classification system based on tumour architecture and with high prognostic impact has been proposed for pulmonary adenocarcinomas (ADCs). For imagingbased prediction of histological ADC subtypes and, thus, prognosis, it is of paramount importance to investigate the correlations of radio- and histomorphological parameters.

Associations between histomorphological ADC growth patterns (lepidic, acinar, papillary, micropapillary and solid) and data from pre-operative assessment by computed tomography (CT) imaging of 174 resected pulmonary ADCs were analysed.

Margin configuration as well as solidity/ground glass opacity of an ADC was associated with distinct histomorphological ADC growth patterns. Solid-predominant ADCs usually had smooth margins and were also solid in CT scans, while lepidic-predominant ADCs had no predominant margin pattern, were located in the periphery, showed a positive bronchogram and were frequently associated with solidity/ground glass opacity. In addition, nonspherical tumour growth was a negative predictor of overall and disease-specific patient survival.

We defined CT morphological parameters that were associated with histomorphological growth patterns of pulmonary ADCs. These data may form the basis for the development of future prognostic algorithms in the palliative setting, which include an integrated evaluation of biopsy histomorphology and CT scan morphology of nonresectable pulmonary ADC.

KEYWORDS: Histology, prediction, prognosis, pulmonary adenocarcinoma, radiology, subtyping

ung cancer is a major cause of cancerrelated morbidity and mortality throughout the world [1]. The most prevalent type of lung cancer is, in $\sim 50 \%$ of cases, pulmonary adenocarcinoma (ADC). This makes pulmonary ADC in itself the leading cause of cancer-related death in many countries.

On the basis of a wealth of recent data showing that histomorphological growth patterns are associated with survival differences [2-12], a multidisciplinary expert panel of the International Association for the Study of Lung Cancer (IASLC), the American Thoracic Society (ATS) and the European Respiratory Society (ERS) proposed a classification system for pulmonary ADC including major paradigm shifts [13], which relies on the tumour architecture and is intended to allow for standardised tumour grading [14]. The prognostic impact of this classification has been confirmed in ADCs of various stages $[9,10,12]$. In preceding studies we could demonstrate that this classification system is a stage-independent predictor of overall (OS), disease-specific (DSS) and disease-free survival (DFS) [15], and is applicable with reasonable interobserver agreement [16]. Additionally, for the first time, we provided evidence that specific growth patterns might have impact on the outcome under adjuvant radio- and chemotherapeutic strategies [15]. However, all these data have been gathered by the use of resection specimens.

As $\sim 70 \%$ of nonsmall cell lung cancer are not resectable at the point of diagnosis, late-stage patients may benefit from an imaging-based ADC subtyping approach in the palliative setting, ideally in combination with histological subtype assessment on biopsies. Comparison of radiographic and morphological patterns might indicate whether the biopsy taken is representative for

Earn CME accreditation by answering questions about this article. You will find these at the back of the printed copy of this issue or online at www.erj.ersjournals.com/misc/cmeinfo.xhtml

This article has supplementary material available from www.erj.ersjournals.com

AFFILIATIONS

*Dept of Radiology, University Hospital Heidelberg, Heidelberg,

\#Dept of Interventional and Diagnostic Radiology with Nuclear Medicine, Thoraxklinik Heidelberg, Heidelberg University, Heidelberg,

${ }^{+}$Translational Research Unit,

Thoraxklinik Heidelberg, Heidelberg University, Heidelberg,

${ }^{\S}$ Institute of Pathology, University Hospital Heidelberg, Heidelberg,

${ }^{f}$ Dept of Pneumology and Critical Care Medicine, Thoraxklinik Heidelberg, Heidelberg University, Heidelberg, and

**Dept of Thoracic Surgery,

Thoraxklinik Heidelberg, Heidelberg University, Heidelberg, Germany.

-University Hospital of Bordeaux,

Bordeaux, France.

\#\#These authors contributed equally.

CORRESPONDENCE

A. Warth

Institute of Pathology

University Hospital Heidelberg

Im Neuenheimer Feld 224

D-69120 Heidelberg

Germany

E-mail: arne.warth@med.uni-

heidelberg.de

Received:

April 052012

Accepted after revision:

June 252012

First published online:

July 262012 
the whole tumour and may indicate the possible necessity of further biopsies of different locations. Furthermore, pattern changes during treatment as a result of clonal selection might be monitored by the evaluation of computed tomography (CT) morphology. In addition, imaging-based data of ADC subtypes may help thoracic surgeons to determine optimised resection strategies. For example, in contrast to solid- or micropapillarypredominant ADC, lepidic predominant ADC [17] could, in the future, eventually be treated with tissue-sparing approaches due to the much better outcome and the scarcity of nodal metastases in this ADC subtype [9, 10, 12, 15].

In order to provide a basis for imaging-based subtyping and stratification of pulmonary ADC, multislice CT-derived characteristics were correlated to the individual histomorphology of resected pulmonary ADCs classified according to the IASLC/ATS/ERS system [14].

\section{PATIENTS AND METHODS}

A previously described, clinically and histopathologically wellcharacterised cohort [15] of 500 completely resected pulmonary ADCs classified according to the novel IASLC/ATS/ERS system was used in our study. Based on this cohort, our picture archiving and communication system (PACS) was screened for available pre-operative multislice CT scans. This resulted in 174 evaluable cases. All patients were treated at a single centre between 2002 and 2008. None of the patients received neoadjuvant chemotherapy. The seventh edition of the International Union Against Cancer/American Joint Committee on Cancer tumour-node-metatstasis (TNM) classification was applied to all cases, resulting in the following number of cases per tumour stage: IA, $n=32 ;$ IB, $n=52$; IIA, $n=19 ;$ IIB, $n=11$; IIIA $n=50$; IIIB, $\mathrm{n}=1$; and IV, $\mathrm{n}=9$. OS, DSS and DFS were recorded. Mean follow-up was 48.5 months.

\section{Morphological evaluation}

Morphology of all cases was evaluated previously [15]. We determined the overall mean percentage of histological patterns. To do so, the percentages of a given pattern were noted for all cases and the mean percentage was calculated for each of the different patterns. This measure was used to compare frequencies of patterns in differing CT-morphological characteristics.

Next, a predominant pattern was assigned to every case as previously described [15]. This measure has been shown to be of prognostic relevance [15]. Within the current article, this data has been used only as an inclusion parameter in the multivariate survival analysis.

\section{CT imaging}

CT scans were performed using either a four-detector (Somatom Volume Zoom; Siemens Medical Solutions, Erlangen, Germany) or a 16-detector (Somatom Sensation 16; Siemens) system. Data were obtained at full inspiration with contrast media administration (Imeron 300; Bracco, Milan, Italy) using the following parameters: $120-\mathrm{kV}$ tube voltage, $210-\mathrm{mA} \cdot \mathrm{s}^{-1}$ (range 110$270 \mathrm{~mA} \cdot \mathrm{s}^{-1}$ ) tube current and $1.25-2.5-\mathrm{mm}$ collimation. Image data were reconstructed using both standard (B40f) and high resolution algorithm (B70f), 2.5-mm section thickness, 2.5-mm reconstruction interval, a typical field of view of $380 \mathrm{~mm}$ and a $512 \times 512$ matrix. Data were transferred to a PACS (Synapse; Fuji Medical System, Tokyo, Japan). All examination datasets were read using both mediastinal (width, $360 \mathrm{HU}$; level, $40 \mathrm{HU}$ ) and lung (width, $1600 \mathrm{HU}$; level, -600 HU) window images at a calibrated PACS workstation allowing for typical interactive reporting.

Two chest radiologists (M. Lederlin and M. Puderbach, with 6 and 9 years experience in chest $\mathrm{CT}$, respectively), blinded to the clinical and histological findings, assessed CT scans retrospectively. Decisions on tumour characteristics were reached by consensus. Referring to previously published work [18-27], characteristics such as tumour shape, sphericity, location, margins and attenuation were recorded (fig. 1). Shapes were classified as round, ovoid, bilobed or irregular. Sphericity was assessed qualitatively. Tumour location (central or peripheral) was also recorded. Margin characteristics were classified as smooth, spiculated, lobulated or mixed, i.e. without a clearly predominant pattern. Each tumour was classified as solid, semisolid or pure ground glass opacity (GGO). When semi-solid, the percentage of GGO was classified as follows: 0-25\%, 25-50\%, $50-75 \%$ and $75-100 \%$. The presence of internal air bronchogram or cavitation within the tumour was additionally recorded.

\section{Statistical analyses}

The continuous percentages of histological growth patterns in dependence of categorical radiological features were compared by either two-sided t-test or one-way ANOVA. Differences in OS, DSS and DFS were computed by Kaplan-Meier analysis and a log rank test was used to probe for statistical significance. Multivariate survival analyses were performed by the Cox proportional hazards model. $\mathrm{p}$-values $<0.05$ were considered significant.

\section{RESULTS}

\section{Frequency of histomorphological and radiomorphological characteristics}

Morphology

Overall the acinar pattern was the most frequently observed histological growth pattern with a mean percentage of $40 \%$, followed by solid (32\%), lepidic (13\%) micropapillary $(8.0 \%)$ and papillary $(7 \%)$.

\section{Radiology}

For the assessment of radiological features, we classified all cases according to the predominance of a specific characteristic in a given category. With respect to radiological shape, the most frequent configuration was round (68 cases, 39.1\%), followed by irregular (62 cases, $35.6 \%$ ), ovoid (37 cases, $21.3 \%$ ) and bilobed (seven cases, $4 \%$ ). The most predominant radiological margin configuration was spiculated (77 cases, 44.3\%), followed by lobulated (51 cases, $29.3 \%$ ), smooth ( 25 cases, $14.4 \%$ ) and mixed (21 cases, 12\%). All distributions of the specific characteristics are given in table 1 .

With regard to the radiological evaluation of attenuation features, most tumours were solid (109 cases, 62.7\%), while only two $(1.1 \%)$ tumours showed a GGO configuration. The remaining $63(36.2 \%)$ cases were categorised as being semi-solid.

Of our cohort, $87(50 \%)$ cases showed an internal air bronchogram by $\mathrm{CT}$, while $12(6.9 \%)$ cases were cavitated and $73(42 \%)$ cases showed none of these two characteristics 

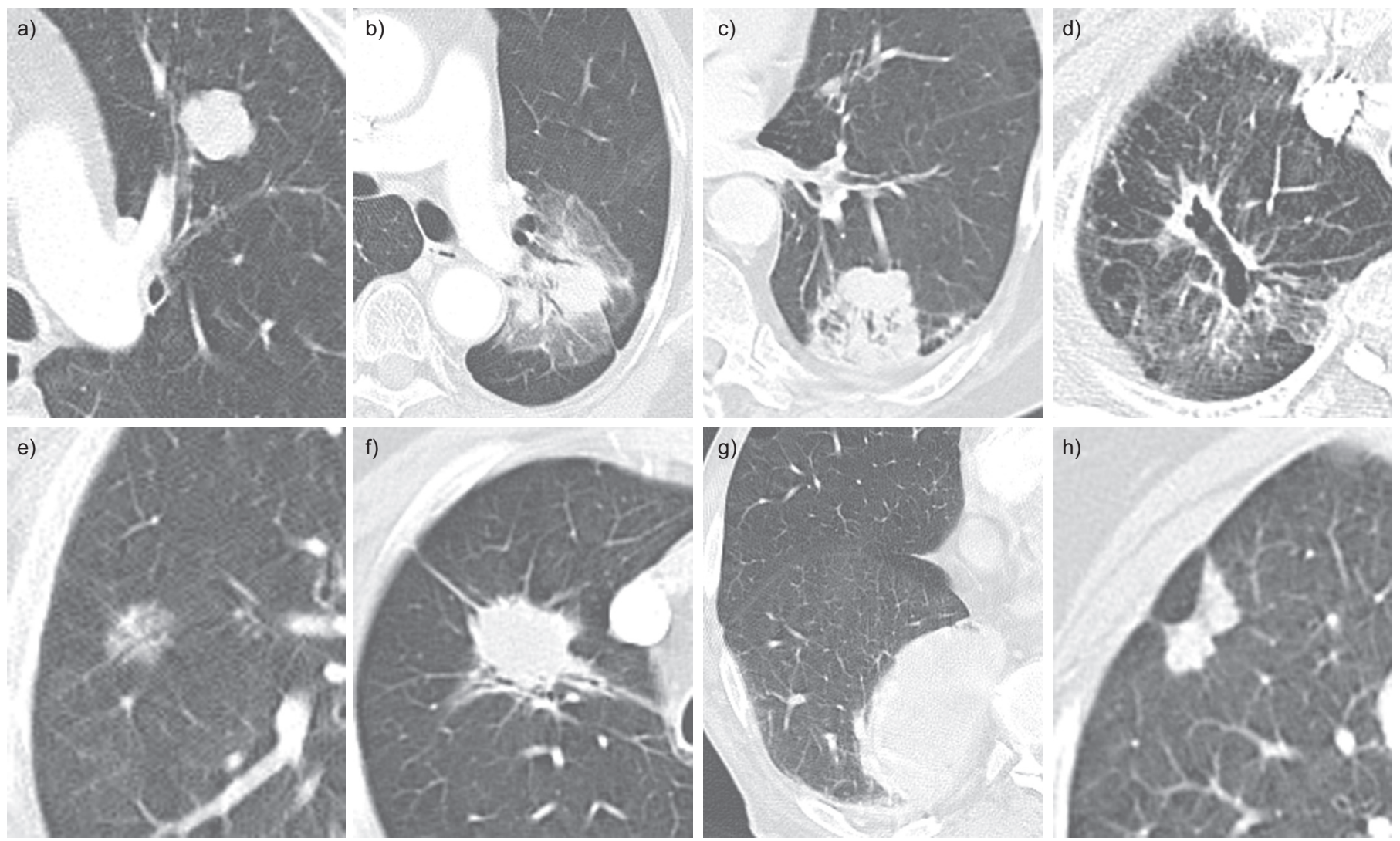

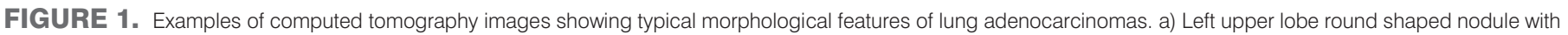

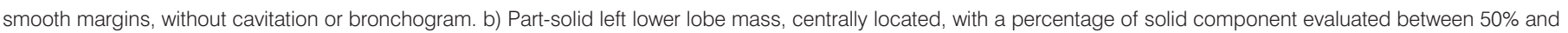

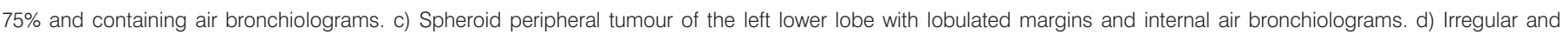

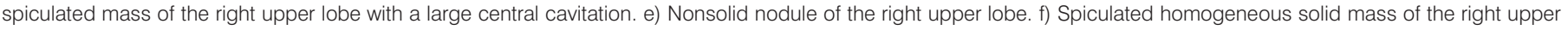

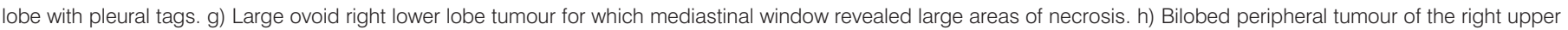
lobe with partly lobulated, spiculated and concave margins.

(table 1). These features were not applicable to the two $(1.1 \%)$ tumours consisting of pure GGO.

\section{Correlation of CT parameters}

When correlating CT parameters with each other, we found significant associations between the different radiomorphological characteristics that might be helpful for CT-based subtyping. Sphericity was significantly associated with characteristics of tumour shape. Furthermore, besides others, attenuation was associated with round/nonround and regular/irregular tumour shape, but also with specific characteristics of the predominant margin type (smooth/nonsmooth and spiculated/nonspiculated). All respective data are given in table S1.

\section{Growth patterns versus sphericity and shape}

ADC with spherical and nonspherical configuration showed comparable overall percentages for all growth patterns (table 1). The same was true for radiological tumour shape, and this characteristic also did not correlate with histomorphological growth patterns (table 1).

\section{Growth patterns versus tumour location}

By studying the influence of tumour location, we found that peripheral ADCs had a significantly higher percentage of lepidic growth pattern than central ADCs $(15.2 \%$ versus $6.4 \%$, $\mathrm{p}=0.023$ ) (table 1). The other histological growth patterns did not correlate with tumour location.

\section{Growth patterns versus CT-based tumour margins}

The occurrence of the respective CT-derived tumour margin classifiers was correlated with the percentages of histomorphological growth patterns (table 1). The occurrence of smooth contours was not significantly associated with the occurrence of any pattern; the percentage of solid growth was slightly higher in those ADC with partially smooth margins $(38.7 \%$ versus $29.0 \% ; p=0.118$ ) (table 1 ). In contrast, those tumours with partially spiculated margins were significantly less likely to contain the lepidic pattern $(p=0.018)$. Lobulated margins did not correlate with any specific histotype (table 1).

Next, CT tumour margins were condensed to a predominant margin type and correlated to histomorphological growth patterns (table 1). Those ADCs with predominant smooth margins showed the highest solid growth percentage $(57.8 \%)$, which was significantly higher $(\mathrm{p}<0.001)$ than the percentages of solid growth in those ADC with predominant spiculated (30.9\%), predominant lobulated $(27.4 \%)$ or mixed margins $(15.2 \%)$. Lepidic growth was significantly more likely to occur in tumours with mixed margins $(27.4 \%)$ than in tumours with predominant 


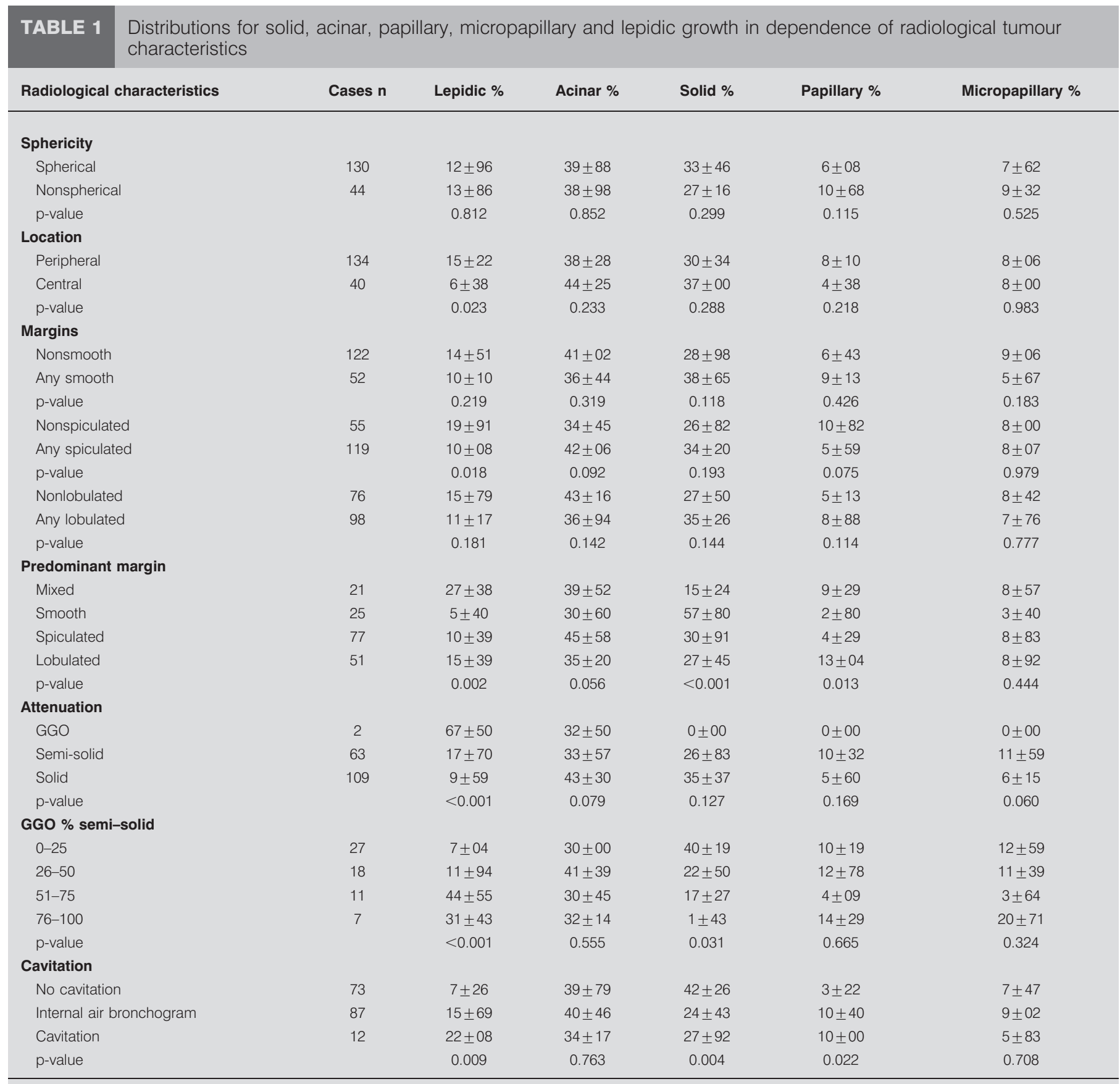

Data are presented as mean \pm SD, unless otherwise stated. GGO: ground-glass opacity.

spiculated $(10.4 \%)$ or predominant smooth margins $(5.4 \%$; $\mathrm{p}=0.002$ ) (table 1 ). The percentage of papillary growth also differed significantly with respect to the predominant margin configuration $(p=0.013)$ (table 1$)$, but the rather low percentages of papillary growth in this cohort probably renders the data not applicable for clinical routine yet. The occurrence of micropapillary and acinar patterns were not significantly associated with any predominant margin configuration.

Taken together, CT-based predominant tumour margins were identified as suitable imaging characteristics with which to determine the predominant ADC histotype.

\section{Growth patterns versus CT attenuation}

Tumours with a solid appearance at CT were more likely to contain solid and acinar growth in histology (43.3\% and $35.4 \%)$, when compared to semi-solid (33.6\% and $26.8 \%)$ and GGO tumours $(32.5 \%$ and $0 \%)$, respectively (acinar: $p=0.079$, solid: $\mathrm{p}=0.127$ ) (table 1 ). In contrast, lepidic growth was significantly less likely $(\mathrm{p}<0.001)$ to occur in solid tumours as determined by CT $(9.6 \%)$ when compared with semi-solid (17.7\%) and GGO $(67.5 \%)$ tumours (table 1$)$. Conversely, solid growth was significantly $(\mathrm{p}=0.031)$ (table 1$)$ more frequent in tumours with lower GGO content, while lepidic growth was significantly 
$(\mathrm{p}<0.001)$ more prevalent in tumours with high GGO content (table 1).

\section{Growth patterns versus cavitation and bronchogram}

Lepidic as well as papillary tumour growth was rare in purely solid ADC without internal air bronchogram or cavitation in CT $(p=0.009$ and $p=0.022$, respectively). Tumours that were solid in histomorphology were usually also solid and noncavitated in CT scans. Micropapillary and acinar differentiation did not differ with respect to bronchogram or cavitation (table 1).

\section{Imaging characteristics versus survival}

Overall, tumour shape was not significantly associated with OS $(p=0.643)$, DSS $(p=0.477)$ or DFS $(p=0.222)$ (fig. S1). In contrast, spherical ADC showed a significantly better OS (68.7 versus 52.4 months, $\mathrm{p}=0.020$ ) and DSS (82.4 versus 59.6 months, $\mathrm{p}=0.030$ ), but not DFS (53.5 versus 50.3 months, $\mathrm{p}=0.399$ ) as compared with nonspherical ADC (fig. 2 and table 2). It is noteworthy that there was a clear link between sphericity and anatomical location of the tumours; the percentage of peripheral location for spherical and nonspherical tumours was $81 \%$ and $66 \%(p=0.043)$, respectively.

There was no significant association between tumour margins and survival; however, predominantly spiculated ADCs had a better DFS (borderline significant) than other ADCs (table 2 and fig. S1).

ADCs with pure GGO attenuation had a $100 \%$ OS, DSS and DFS; however, this group consisted of only two cases in our cohort. In CT scans, semi-solid and solid ADCs showed no striking difference in OS and DSS, and a reduced but statistically nonsignificant ( $\mathrm{p}=0.087$ ) DFS was evident for solid ADCs (47.7 months) compared with semi-solid tumours (63.4 months) (fig. 3). However, in semi-solid neoplasms, an association of the percentage of GGO with OS, DSS and DFS could not be established.

Finally, we tested whether the impact of sphericity on OS and DSS was an independent one by performing a multivariate survival analysis under inclusion of sphericity, predominant growth pattern and stage. Here, we found that sphericity failed to show clear-cut independent prognostic impact on OS (hazard ratio (HR) 1.6 for nonspherical versus spherical tumours, $\mathrm{p}=0.066)$, but had stage- and pattern-independent prognostic impact on DSS (HR 2.2, $\mathrm{p}=0.016$ ).

\section{DISCUSSION}

This is the first study to correlate radiomorphological features with histomorphological characteristics of resected invasive pulmonary ADC according to the novel IASCL/ATS/ERS classification [14]. We performed this study to establish criteria for imaging-based assessment of pulmonary ADC growth patterns. Such growth pattern evaluation, specifically the separation of lepidic predominant (good prognosis) from acinar predominant (moderate prognosis) and micropapillary or solid predominant ADC (poor prognosis) has been shown to be of utmost prognostic importance.

We demonstrate that CT morphological features might provide promising clues to predict histological growth patterns and patient survival. Notably, CT features did not exhibit the same degree of association with histomorphology in our study. For instance, unlike tumour margins, attenuation or cavitation, neither shape nor sphericity correlated with any ADC growth pattern. In addition, whereas histological grading of ADC was shown to be an efficient discriminator for patient prognosis [15], only few CT parameters showed prognostic significance in the present study. Therefore, CT features alone do not seem to be sufficient in the palliative setting where histomorphology-based analyses of the whole tumour can obviously not be performed. In those patients, the combination of individual tumour characteristics as derived from the morphology of a limited biopsy sample in conjunction with well-focused CT morphological features of the whole tumour might be considered for a more detailed tumour assessment.

The strong association between GGO and lepidic growth, and between CT solidity and solid growth are important results of this study. Beyond geometrical measurements, CT offers the ability of tissue attenuation measurements. In 2002, the terms of solid (i.e. completely obscuring the lung parenchyma within it),
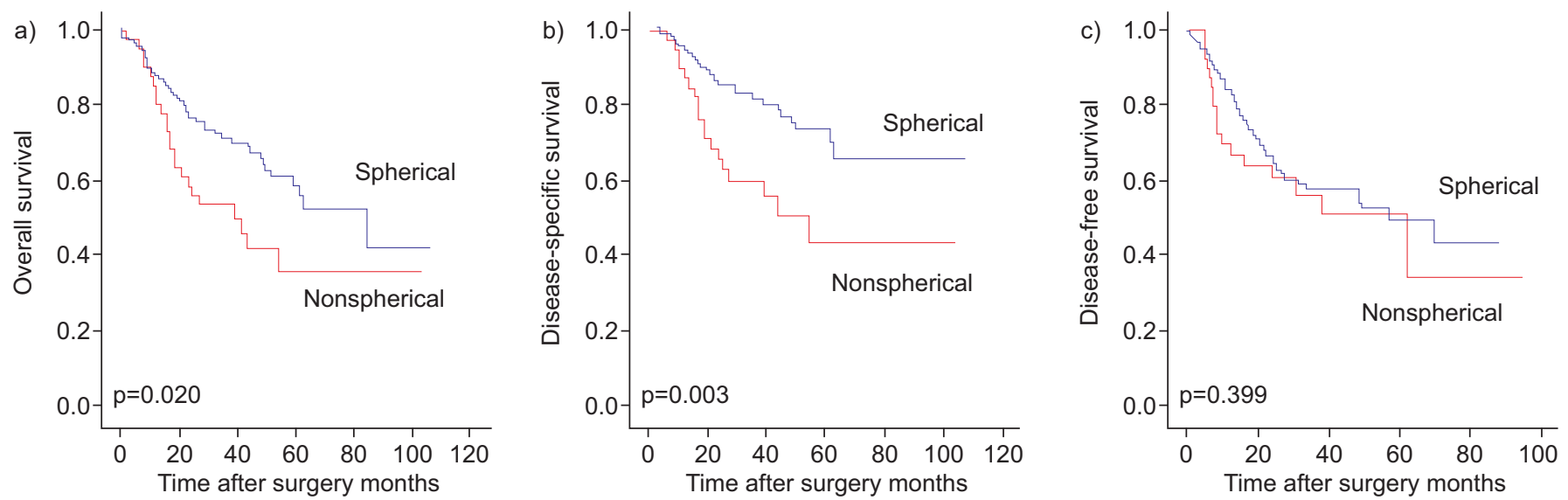

FIGURE 2. Association of tumour sphericity with survival. The differences for a) overall, b) disease-specific and c) disease-free survival in dependence of tumour sphericity as determined by computed tomography are depicted. 


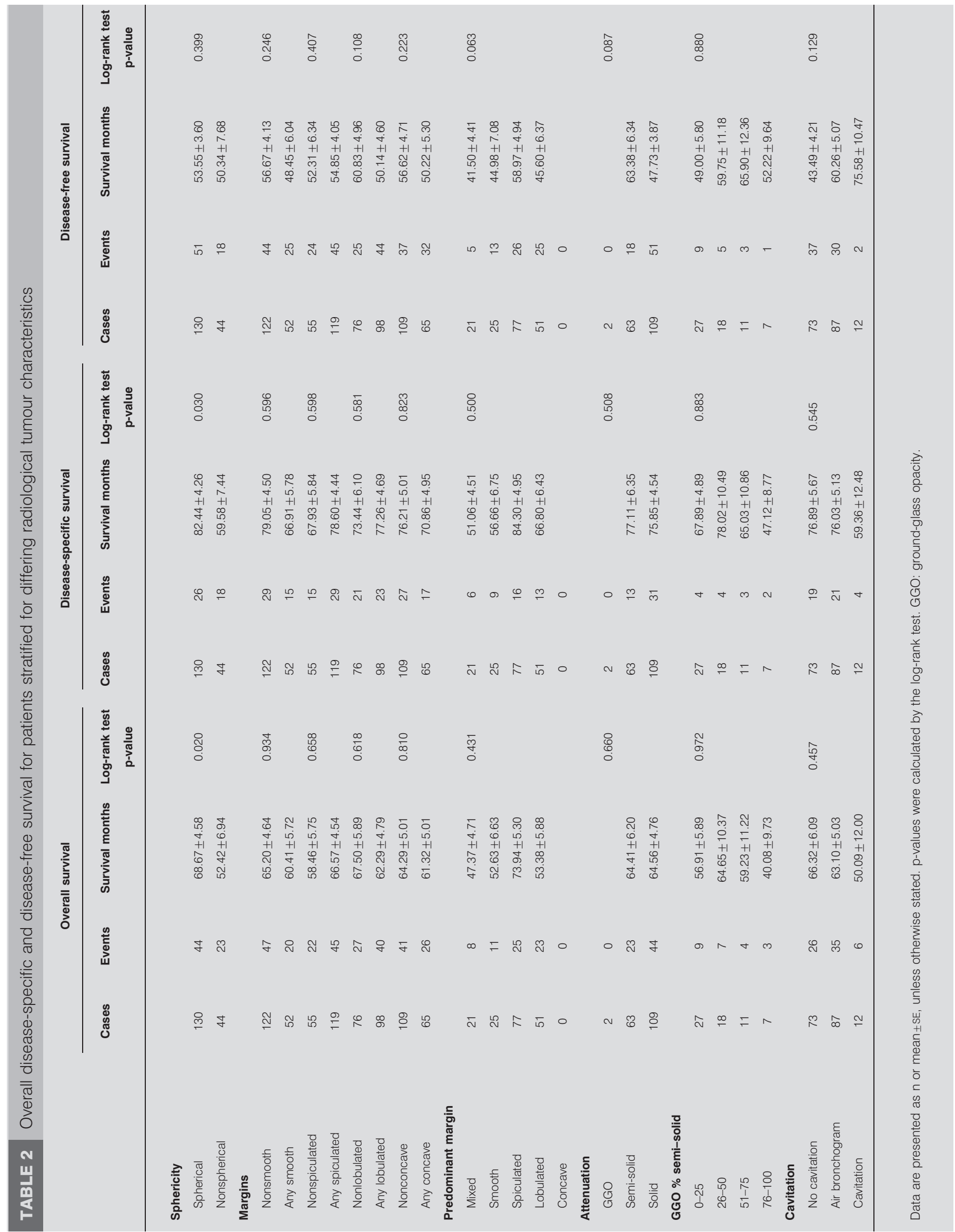



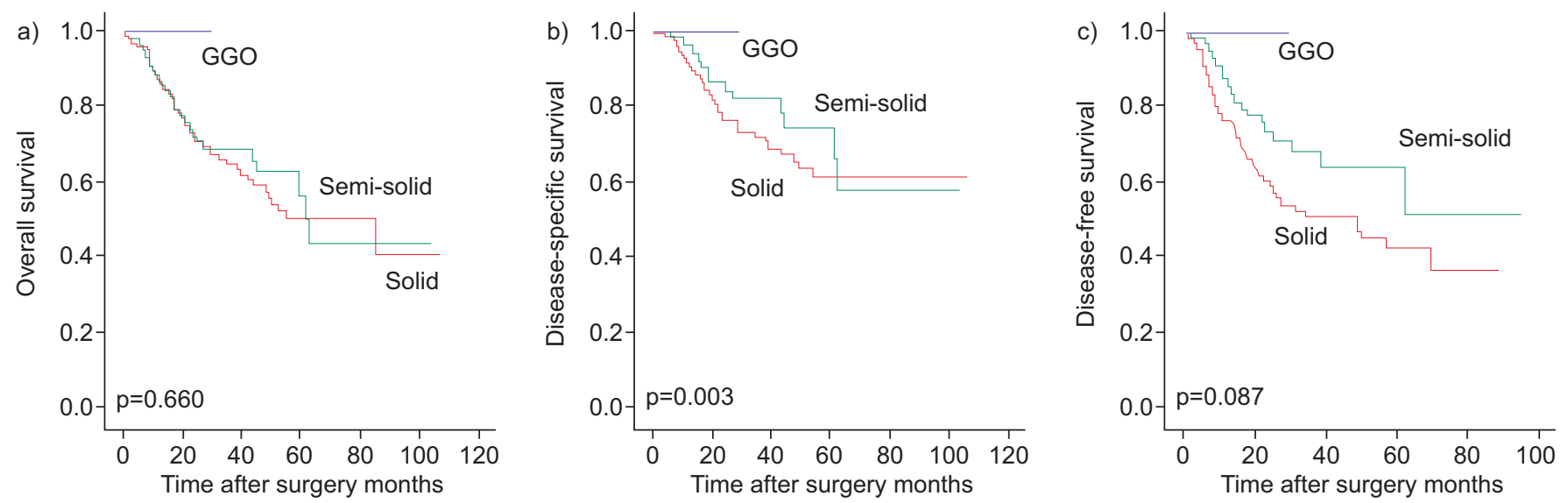

FIGURE 3. Association of tumour computed tomography (CT) attenuation with survival. The differences for a) overall, b) disease-specific and c) disease-free survival in dependence of attenuation of the lesions as determined by CT are depicted. GGO: ground-glass opacity.

part-solid and nonsolid (i.e. pure GGO without any solid part) lung nodules were introduced [28]. Invasive ADCs usually display a solid or part-solid appearance and rarely a pure GGO aspect [25, 29], which is also confirmed by our study. Furthermore, low-grade ADCs have been found to be predominantly part-solid or nonsolid tumours [18, 19, 22, 27], which is also in line with our data. Specifically, there is a growing body of evidence that GGO appearance correlates with the occurrence of the lepidic growth pattern [24, 30-33]. In a previous study, VASQUEZ et al. [34] showed that the proportion of this ADC growth pattern represented a positive prognostic factor and correlated with the proportion of GGO at CT. In our study, we could confirm this by demonstrating a significant association between the percentage of the GGO component and the mean percentage of lepidic growth in the respective tumours. These results suggest that the percentage of GGO could be considered as a radiological surrogate for lepidic growth, thereby allowing a comprehensive pre-operative assessment in this regard. As suggested, in the palliative setting with positive biopsy for ADC, the percentage of GGO might be used as an indicator of a potentially better prognosis, as is also corroborated by the near significant difference in DFS between semi-solid and solid ADC. This is further supported by the inverse correlation between the percentage of GGO and solid growth pattern, as the solid growth pattern is associated with a poor prognosis [15, 35].

We paid careful attention to note and document small foci of consolidation within GGO areas. In doing so, only two ADCs out of 174 displayed a pure GGO appearance, while all other tumours were classified as part-solid.

It must also be noted that the correlation between lepidic growth and the percentage of GGO was by no means perfect, which is certainly due, in part, to the fact that peripheral GGO in semi-solid tumours might also represent reactive conditions including peritumoral inflammation, oedema, fibrosis or organising pneumonia $[20,36]$.

Besides attenuation, other morphological CT features are likely to offer additional information towards a more detailed and comprehensive assessment of the quantity of noninvasive ADC tumour components. Peripheral ADCs displayed more lepidic growth pattern than central ADCs (table 1), which is concordant with the generally better survival rate of subjects with peripheral ADCs.

The link between spheroid configuration and peripheral location (table S1) may at least in part account for the better OS and DSS of spheroid ADCs, as those tumours are less likely to involve mediastinal or hilar structures.

Similarly to the IASLC/ATS/ERS classification, which is based on the predominant histological pattern, CT analysis of ADC margins was of particular interest when focusing on the predominant margin type. Many studies have shown that spiculated and lobulated edges are suggestive of malignancy [37-39]. However, a predominantly smooth nodule is usually related to either a benign lesion, such as hamartoma, or metastasis in patients with a history of malignancy [40]. If such a smooth nodule turns out to be a pulmonary ADC, our results indicate that this tumour is likely to have a higher percentage of solid growth and thus a worse outcome compared with ADC with other predominant margin types. To our knowledge, the association between smooth margins and this high-grade pattern has not been reported so far. These data are also supported by the inverse association between smooth margins and lepidic growth, as well as the worse DSS of smooth-margin ADC (57 months) as compared with spiculated (84 months) and lobulated ADC (67 months). Furthermore, the spiculated CT pattern showed an inverse association with the lepidic growth pattern. Spiculations and associated features, such as vascular convergence and pleural tags, have been shown to be related to the development of central fibrosis within the tumour resulting in tissue contraction [7, 41] and indicating invasive tumour growth. These changes might occur in ADC undergoing stepwise progression towards invasiveness when initial lepidic architecture transforms into acinar or (micro-)papillary, and finally solid growth. Nevertheless, we did not find any significant difference in survival rates between spiculated and nonspiculated ADC.

It is worth noting that predominantly lobulated ADC contained more papillary and micropapillary growth patterns (the two rarest patterns) than spiculated and smooth ADC. To date, lobulated margins were only known as reliable CT features to 
predict malignancy by indicating uneven growth rates with foci of malignant cells at the periphery of the tumour [42].

Internal air bronchogram or bronchiologram has been assigned to airways distortion due to a desmoplastic reaction and has been reported in up to $55 \%$ of lepidic predominant ADCs $[25,43]$. In line with this data, the occurrence of an air bronchogram and bronchiologram was positively associated with the lepidic growth pattern and negatively associated with the solid growth pattern in our study.

As well as CT, magnetic resonance imaging (MRI) has also been investigated for the characterisation of lung cancers. This included novel MRI techniques with diffusion-weighted imaging (DWI), which have been used for the differentiation of benign and malignant lung lesions [44, 45]. However, in one study DWI failed to differentiate the lepidic subtype from other growth patterns [46]. Nevertheless, significant correlations between MRI diffusion evaluation and survival have been reported [47], indicating a promising role for this imaging approach in pre-operative tumour characterisation.

One limitation of our study was the restriction to surgical cases, which was necessary because, obviously, only in those cases could a comprehensive histomorphological assessment be performed. Another weakness was that only few lepidic predominant ADCs were included in our study; further studies to validate our findings in larger cohorts of this ADC subtype are warranted.

It was recently suggested that percutaneous biopsies should be restricted to those lesions with solid components measuring $>5 \mathrm{~mm}$ or those measuring more than half a lesion's diameter, i.e. lesions with sufficiently large solid components to allow for a confident biopsy diagnosis of invasive carcinoma [48]. In the light of our results, these criteria might be extended to including features such as location, margins and air bronchogram. These criteria might also be helpful for planning of surgery as well as in CT screening, which has recently been proven to reduce the mortality of lung cancer in heavy smokers (20\%) as compared with radiographic screening [49].

In conclusion, specific CT features analysed in this study were predictive of the histomorphological tumour growth patterns of pulmonary ADC as defined by the new IASLC/ATS/ERS classification [14]. By providing a more detailed noninvasive assessment, CT features might be used to build stratification algorithms for the palliative setting. The prerequisite for setting up such decisional algorithm is a thorough evaluation of the reproducibility of CT features, which should be tested in future studies. One could imagine an algorithm including tumour location, sphericity, smooth or spiculated margins, percentage of GGO and air bronchogram to separate pulmonary ADCs with good prognosis from those with poor prognosis. Such an algorithm, especially in conjunction with biopsy data, may improve the management of part-solid nodules, which remains a critical matter.

Prospectively, we believe that combined radiomorphological and histomorphological efforts might play a significant role in ADC subtyping and respective algorithms might ultimately contribute to refine patient treatment stratifications and consequently an improved outcome.

\section{SUPPORT STATEMENT}

This study was supported by the LungSys consortium and the German Centre for Lung Research (Deutsches Zentrum für Lungenforschung and Deutsches Zentrum für Lungenforschung). M. Lederlin was supported by the French Society of Radiology.

\section{STATEMENT OF INTEREST}

Conflict of interest information can be found alongside the online version of this article at www.erj.ersjournals.com

\section{ACKNOWLEDGEMENTS}

M. Puderbach, T. Mulcy, P.A. Schnabel, H.-U. Kauczor, C.P. Heussel, F.J.F. Herth and H. Dienemann are members of the German Center for Lung Research (DZL).

\section{REFERENCES}

1 Jemal A, Siegel R, Xu J, et al. Cancer statistics, 2010. CA Cancer J Clin, 60: 277-300.

2 Amin MB, Tamboli P, Merchant SH, et al. Micropapillary component in lung adenocarcinoma: a distinctive histologic feature with possible prognostic significance. Am J Surg Pathol 2002; 26: 358-364.

3 Barletta JA, Yeap BY, Chirieac LR. Prognostic significance of grading in lung adenocarcinoma. Cancer 2010; 116: 659-669.

4 Borczuk AC, Qian F, Kazeros A, et al. Invasive size is an independent predictor of survival in pulmonary adenocarcinoma. Am J Surg Pathol 2009; 33: 462-469.

5 Miyoshi T, Satoh Y, Okumura S, et al. Early-stage lung adenocarcinomas with a micropapillary pattern, a distinct pathologic marker for a significantly poor prognosis. Am J Surg Pathol 2003; 27: 101-109.

6 Motoi N, Szoke J, Riely GJ, et al. Lung adenocarcinoma: modification of the $2004 \mathrm{WHO}$ mixed subtype to include the major histologic subtype suggests correlations between papillary and micropapillary adenocarcinoma subtypes, EGFR mutations and gene expression analysis. Am J Surg Pathol 2008; 32: 810-827.

7 Noguchi M, Morikawa A, Kawasaki M, et al. Small adenocarcinoma of the lung. Histologic characteristics and prognosis. Cancer 1995; 75: 2844-2852.

8 Riquet M, Foucault C, Berna P, et al. Prognostic value of histology in resected lung cancer with emphasis on the relevance of the adenocarcinoma subtyping. Ann Thorac Surg 2006; 81: 1988-1995.

9 Russell PA, Wainer Z, Wright GM, et al. Does lung adenocarcinoma subtype predict patient survival? A clinicopathologic study based on the new International Association for the Study of Lung Cancer/American Thoracic Society/European Respiratory Society international multidisciplinary lung adenocarcinoma classification. J Thorac Oncol 2011; 6: 1496-1504.

10 Sica G, Yoshizawa A, Sima CS, et al. A grading system of lung adenocarcinomas based on histologic pattern is predictive of disease recurrence in stage I tumors. Am J Surg Pathol 2010; 34: $1155-1162$.

11 Yim J, Zhu LC, Chiriboga L, et al. Histologic features are important prognostic indicators in early stages lung adenocarcinomas. Mod Pathol 2007; 20: 233-241.

12 Yoshizawa A, Motoi N, Riely GJ, et al. Impact of proposed IASLC/ ATS/ERS classification of lung adenocarcinoma: prognostic subgroups and implications for further revision of staging based on analysis of 514 stage I cases. Mod Pathol 2011; 24: 653-664.

13 Travis WD, Brambilla E, Van Schil P, et al. Paradigm shifts in lung cancer as defined in the new IASLC/ATS/ERS lung adenocarcinoma classification. Eur Respir J 2011; 38: 239-243.

14 Travis WD, Brambilla E, Noguchi $M$, et al. International Association for the Study of Lung Cancer/American Thoracic Society/European Respiratory Society international multidisci- 
plinary classification of lung adenocarcinoma. J Thorac Oncol 2011; 6: 244-285.

15 Warth A, Muley T, Meister M, et al. The novel histologic IASLC/ ATS/ERS classification of invasive pulmonary adenocarcinoma is a stage-independent predictor of survival. J Clin Oncol 2012; 30: 1438-1446.

16 Warth A, Stenzinger A, von Brunneck AC, et al. Interobserver variability in the application of the novel IASLC/ATS/ERS classification. Eur Respir J 2012; 40: 1221-1227.

17 Dadrich M, Schneider T, Puderbach M, et al. Pleuraherd auf Wanderschaft. [Moving pleural mass]. Med Klin Intensivmed Notfmed 2012; 107: 213-215.

18 Aoki T, Nakata H, Watanabe $\mathrm{H}$, et al. Evolution of peripheral lung adenocarcinomas: CT findings correlated with histology and tumor doubling time. AJR Am J Roentgenol 2000; 174: 763-768.

19 Aoki T, Tomoda Y, Watanabe H, et al. Peripheral lung adenocarcinoma: correlation of thin-section CT findings with histologic prognostic factors and survival. Radiology 2001; 220: 803-809.

20 Gandara DR, Aberle D, Lau D, et al. Radiographic imaging of bronchioloalveolar carcinoma: screening., patterns of presentation and response assessment. J Thorac Oncol 2006; 1: Suppl. 9, S20-S26.

21 Godoy MC, Naidich DP. Subsolid pulmonary nodules and the spectrum of peripheral adenocarcinomas of the lung: recommended interim guidelines for assessment and management. Radiology 2009; 253: 606-622.

22 Kuriyama K, Seto M, Kasugai T, et al. Ground-glass opacity on thin-section CT: value in differentiating subtypes of adenocarcinoma of the lung. AJR Am J Roentgenol 1999; 173: 465-469.

23 Li F, Sone S, Abe H, et al. Malignant versus benign nodules at CT screening for lung cancer: comparison of thin-section CT findings. Radiology 2004; 233: 793-798.

24 Suzuki K, Kusumoto M, Watanabe S, et al. Radiologic classification of small adenocarcinoma of the lung: radiologic-pathologic correlation and its prognostic impact. Ann Thorac Surg 2006; 81: 413-419.

25 Zwirewich CV, Vedal S, Miller RR, et al. Solitary pulmonary nodule: high-resolution $\mathrm{CT}$ and radiologic-pathologic correlation. Radiology 1991; 179: 469-476.

26 Lee HJ, Goo JM, Lee CH, et al. Predictive CT findings of malignancy in ground-glass nodules on thin-section chest CT: the effects on radiologist performance. Eur Radiol 2009; 19: 552-560.

27 Takashima S, Maruyama Y, Hasegawa M, et al. CT findings and progression of small peripheral lung neoplasms having a replacement growth pattern. AJR Am J Roentgenol 2003; 180: 817-826.

28 Henschke CI, Yankelevitz DF, Mirtcheva R, et al. CT screening for lung cancer: frequency and significance of part-solid and nonsolid nodules. AJR Am J Roentgenol 2002; 178: 1053-1057.

29 Travis WD, Garg K, Franklin WA, et al. Evolving concepts in the pathology and computed tomography imaging of lung adenocarcinoma and bronchioloalveolar carcinoma. J Clin Oncol 2005; 23: 3279-3287.

30 Kodama K, Higashiyama M, Yokouchi H, et al. Prognostic value of ground-glass opacity found in small lung adenocarcinoma on high-resolution CT scanning. Lung Cancer 2001; 33: 17-25.

31 Seki N, Sawada S, Nakata M, et al. Lung cancer with localized ground-glass attenuation represents early-stage adenocarcinoma in nonsmokers. J Thorac Oncol 2008; 3: 483-490.
32 Takashima S, Maruyama Y, Hasegawa M, et al. Prognostic significance of high-resolution CT findings in small peripheral adenocarcinoma of the lung: a retrospective study on 64 patients. Lung Cancer 2002; 36: 289-295.

33 Yang ZG, Sone S, Takashima S, et al. High-resolution CT analysis of small peripheral lung adenocarcinomas revealed on screening helical CT. AJR Am J Roentgenol 2001; 176: 1399-1407.

34 Vazquez M, Carter D, Brambilla E, et al. Solitary and multiple resected adenocarcinomas after CT screening for lung cancer: histopathologic features and their prognostic implications. Lung Cancer 2009; 64: 148-154.

35 Yoshizawa A, Motoi N, Riely GJ, et al. Impact of proposed IASLC/ ATS/ERS classification of lung adenocarcinoma: prognostic subgroups and implications for further revision of staging based on analysis of 514 stage I cases. Mod Pathol 2011; 24: 653-664.

36 Kim HY, Shim YM, Lee KS, et al. Persistent pulmonary nodular ground-glass opacity at thin-section CT: histopathologic comparisons. Radiology 2007; 245: 267-275.

37 Gurney JW, Lyddon DM, McKay JA. Determining the likelihood of malignancy in solitary pulmonary nodules with Bayesian analysis. Part II. Application. Radiology 1993; 186: 415-422.

38 Tang AW, Moss HA, Robertson RJ. The solitary pulmonary nodule. Eur J Radiol 2003; 45: 69-77.

39 Winer-Muram HT. The solitary pulmonary nodule. Radiology 2006; 239: 34-49.

40 Seemann MD, Seemann O, Luboldt W, et al. Differentiation of malignant from benign solitary pulmonary lesions using chest radiography, spiral CT and HRCT. Lung Cancer 2000; 29: 105-124.

41 Shimosato Y, Suzuki A, Hashimoto T, et al. Prognostic implications of fibrotic focus (scar) in small peripheral lung cancers. Am J Surg Pathol 1980; 4: 365-373.

42 Zerhouni EA, Stitik FP, Siegelman SS, et al. CT of the pulmonary nodule: a cooperative study. Radiology 1986; 160: 319-327.

43 Lee KS, Yoon JH, Kim TK, et al. Evaluation of tracheobronchial disease with helical CT with multiplanar and three-dimensional reconstruction: correlation with bronchoscopy. Radiographics 1997; 17: 555-567.

44 Liu $\mathrm{H}$, Liu $\mathrm{Y}, \mathrm{Yu} \mathrm{T}$, et al. Usefulness of diffusion-weighted MR imaging in the evaluation of pulmonary lesions. Eur Radiol 2010; 20: $807-815$.

45 Tondo F, Saponaro A, Stecco A, et al. Role of diffusion-weighted imaging in the differential diagnosis of benign and malignant lesions of the chest-mediastinum. Radiol Med 2011; 116: 720-733.

46 Koyama H, Ohno Y, Aoyama N, et al. Comparison of STIR turbo SE imaging and diffusion-weighted imaging of the lung: capability for detection and subtype classification of pulmonary adenocarcinomas. Eur Radiol 2010; 20: 790-800.

47 Razek AA, Fathy A, Gawad TA. Correlation of apparent diffusion coefficient value with prognostic parameters of lung cancer. J Comput Assist Tomogr 2011; 35: 248-252.

48 Franks TJ, Galvin JR, Jett JR, et al. Expert opinion: role of percutaneous biopsy of part-solid nodules in the IASLC/ATS/ ERS international multidisciplinary classification of lung adenocarcinoma. J Thorac Imaging 2011; 26: 189.

49 Aberle DR, Adams AM, Berg CD, et al. Reduced lung-cancer mortality with low-dose computed tomographic screening. $N$ Engl J Med 2011; 365: 395-409. 